\title{
Predictors of 25(OH)D half-life and plasma 25(OH)D concentration in The Gambia and the UK
}

\author{
K. S. Jones $\cdot$ S. Assar $\cdot$ D. Vanderschueren $\cdot$ R. Bouillon • \\ A. Prentice $\cdot$ I. Schoenmakers
}

Received: 28 April 2014 / Accepted: 12 September 2014 / Published online: 3 October 2014

(C) The Author(s) 2014. This article is published with open access at Springerlink.com

\begin{abstract}
Summary Predictors of $25(\mathrm{OH}) \mathrm{D}_{3}$ half-life were factors associated with vitamin D metabolism, but were different between people in The Gambia and the UK. Country was the strongest predictor of plasma $25(\mathrm{OH}) \mathrm{D}$ concentration, probably as a marker of UVB exposure. $25(\mathrm{OH}) \mathrm{D}_{3}$ half-life may be applied as a tool to investigate vitamin $\mathrm{D}$ expenditure.

Introduction The aim of this study was to investigate predictors of $25(\mathrm{OH}) \mathrm{D}_{3}$ half-life and plasma $25(\mathrm{OH}) \mathrm{D}$ concentration.

Methods Plasma half-life of an oral tracer dose of deuterated$25(\mathrm{OH}) \mathrm{D}_{3}$ was measured in healthy men aged $24-39$ years, resident in The Gambia, West Africa $(n=18)$ and in the UK during the winter $(n=18)$, countries that differ in calcium intake and vitamin D status. Plasma and urinary markers of vitamin $\mathrm{D}$, calcium, phosphate and bone metabolism, nutrient intakes and anthropometry were measured.

Results Normally distributed data are presented as mean (SD) and non-normal data as geometric mean $(95 \% \mathrm{CI})$. Gambian compared to UK men had higher plasma concentrations of 25(OH)D (69 (13) vs. 29 (11) nmol/L; $P<0.0001)$; $1,25(\mathrm{OH})_{2} \mathrm{D}(181(165,197)$ vs. $120(109,132) \mathrm{pmol} / \mathrm{L}$;
\end{abstract}

Ann Prentice and Inez Schoenmakers shared last authorship.

K. S. Jones $(\triangle) \cdot S$. Assar $\cdot$ A. Prentice $\cdot$ I. Schoenmakers Elsie Widdowson Laboratory, Medical Research Council Human Nutrition Research, Fulbourn Road, Cambridge CB1 9NL, UK

e-mail: kerry.jones@mrc-hnr.cam.ac.uk

D. Vanderschueren $\cdot$ R. Bouillon

Clinical and Experimental Endocrinology, Department of Clinical and Experimental Medicine, KU Leuven, Leuven, Belgium

K. S. Jones $\cdot$ A. Prentice

Medical Research Council Keneba, PO BOX 273, Banjul,

The Gambia

D. Vanderschueren $\cdot$ R. Bouillon

Department of Endocrinology, University Hospital, Leuven, Belgium
$P<0.01)$; and parathyroid hormone $(\mathrm{PTH})(50(42,60)$ vs. 33 $(27,39) ; P<0.0001)$. There was no difference in $25(\mathrm{OH}) \mathrm{D}_{3}$ half-life (14.7 (3.5)days vs. 15.6 (2.5)days) between countries $(P=0.2)$. In multivariate analyses, $25(\mathrm{OH}) \mathrm{D}, 1,25(\mathrm{OH})_{2} \mathrm{D}$, vitamin $\mathrm{D}$ binding protein and albumin-adjusted calcium $\left(\mathrm{Ca}_{\mathrm{alb}}\right)$ explained $79 \%$ of variance in $25(\mathrm{OH}) \mathrm{D}_{3}$ half-life in Gambians, but no significant predictors were found in UK participants. For the countries combined, $\mathrm{Ca}_{\mathrm{alb}}$, PTH and plasma phosphate explained $39 \%$ of half-life variability. $1,25(\mathrm{OH})_{2} \mathrm{D}$, weight, PTH and country explained $81 \%$ of variability in $25(\mathrm{OH}) \mathrm{D}$ concentration; however, country alone explained $74 \%$.

Conclusion Factors known to affect 25(OH)D metabolism predict $25(\mathrm{OH}) \mathrm{D}_{3}$ half-life, but these differed between countries. Country predicted $25(\mathrm{OH}) \mathrm{D}$, probably as a proxy measure for UVB exposure and vitamin D supply. This study supports the use of $25(\mathrm{OH}) \mathrm{D}$ half-life to investigate vitamin D metabolism.

Keywords $24,25(\mathrm{OH})_{2} \mathrm{D} \cdot$ Gambia $\cdot$ Half-life $\cdot$ Vitamin D binding protein $\cdot$ Vitamin $D$ metabolism

\section{Introduction}

Vitamin D is essential for human health, and a growing body of evidence links vitamin D status to health outcomes beyond those associated with the musculoskeletal system [1]. Functional biomarkers are required to determine the adequacy of vitamin D status and to determine physiological requirements during growth, pregnancy or ageing and how requirements may be modified by ethnicity, sex, and disease or body composition. Biomarkers are also required to address specific questions such as elucidating the vitamin D-response relationship and its functional outcomes. Plasma 25-hydroxyvitamin $\mathrm{D}(25(\mathrm{OH}) \mathrm{D})$ concentration is the most commonly used marker of vitamin $\mathrm{D}$ status. It is determined by endogenous vitamin D synthesis and dietary intake, conversion into $25(\mathrm{OH}) \mathrm{D}$ and, 
finally, distribution and usage (metabolism and excretion). The concentration of $25(\mathrm{OH}) \mathrm{D}$ in plasma is largely unregulated, and it has a relatively long half-life of 2-3 weeks [2-5].

Plasma 25(OH)D half-life, as a marker of vitamin D usage, may be useful for elucidating the determinants of the vitamin $\mathrm{D}$ dose-response relationship and for examining relationships between vitamin $\mathrm{D}$, calcium, phosphate metabolism and balance, their regulating hormones and other factors related to vitamin D metabolism. In clinical and animal studies that used radiolabelled isotopes, lower plasma $1,25(\mathrm{OH})_{2} \mathrm{D}[6,7]$, lower parathyroid hormone (PTH) [8] and calcium supplementation were associated with a longer half-life [6, 8]. Fibre intake [9] and vitamin D binding protein (DBP) concentration and genotype may also influence $25(\mathrm{OH}) \mathrm{D}$ half-life [10].

We previously described a method to measure $25(\mathrm{OH}) \mathrm{D}$ half-life $[5,10]$ that uses stable isotope tracers. Here, we report an investigation of $25(\mathrm{OH}) \mathrm{D}_{3}$ half-life as a biomarker of $25(\mathrm{OH}) \mathrm{D}$ expenditure by examining relationships between half-life and known drivers of vitamin D metabolism in two distinct populations. The study was performed in healthy men resident in a rural area of The Gambia, West Africa, and in Cambridge, UK, populations known to differ in vitamin D status, calcium intake and markers of vitamin $\mathrm{D}$ metabolism (e.g. $1,25(\mathrm{OH})_{2} \mathrm{D}$ and PTH) [11]. We tested the hypothesis that 25(OH)D half-life in these populations differs due to these differences in dietary calcium intake and vitamin D supply. We also investigated predictors of plasma $25(\mathrm{OH}) \mathrm{D}$ concentration using the same variables.

\section{Subjects and methods}

\section{Study setting}

Detailed descriptions of the study design and subject characteristics were published previously [10]. In short, the study was performed at two centres: MRC Keneba, a rural field station in The Gambia (latitude $13^{\circ} \mathrm{N}$ ), and at MRC Human Nutrition Research (HNR), Cambridge, UK (latitude $52^{\circ} \mathrm{N}$ ). The study was conducted according to the guidelines laid down in the Declaration of Helsinki, and procedures involving the participants were approved by the joint Gambian Government-MRC ethics committee and the UK National Research Ethics Service (NRES) Cambridge committee, respectively. Trained staff explained the study to the participants, and informed written consent was obtained. There is year-round ability to synthesise vitamin D in The Gambia where field work was performed at the end of the dry season, in May and June 2010. In the UK, study procedures were carried out between January and April 2011, when there is little or no cutaneous synthesis of vitamin $\mathrm{D}$ and an exclusion criterion was recent (previous 3 months) or planned (during study period) travel to tropical or subtropical countries or high-altitude areas.
Participants

Participants $(n=36)$ were healthy, nonsmoking males, aged between 25 and 39 years. Exclusion criteria were recent illness (within 2 weeks); a broken bone within the last 3 years; known bone, kidney or liver disease; taking any prescription medicines; or haemoglobin less than $10 \mathrm{~g} / \mathrm{dL}$. All Gambian participants were of Mandinka ethnicity (defined by self-reported ethnicity of the father), and UK subjects were all selfclassified as white.

\section{Study protocols}

The same study protocols were followed in The Gambia and the UK. On day 1 and day 21 of the study, and following an overnight fast and voiding of the first morning urine, a 2-h fasting urine [5] was collected from approximately 7:00 a.m. A blood sample was collected after $1 \mathrm{~h}$. Height (Leicester Stadiometer, Chasmoors Ltd, London, UK), weight (Tanita HD305 scale, Tanita, Amsterdam, The Netherlands) and body composition by bioelectrical impedance (Tanita BC418-MA Tanita, Amsterdam, The Netherlands) were measured. On day 1 , and after completion of the urine collection, participants were given an oral tracer dose of $40 \mathrm{nmol}(16 \mu \mathrm{g})$ of stable isotope-labelled $25(\mathrm{OH}) \mathrm{D}_{3}\left(\mathrm{~d}_{3}-25(\mathrm{OH}) \mathrm{D}_{3}\right)$ (deuterated $(6,19,19)$ 25-hydroxy vitamin $\mathrm{D}_{3}$ ) (product number 705888; 97 atom \%; purity $98 \%$; Sigma-Aldrich, Poole, UK) dissolved in vegetable oil with a standard breakfast [10]. Fasted blood samples were collected into tubes containing lithium heparin anticoagulant on day 6 and ( \pm 2 days) days $9,21,24,27,30$ and 33 for measurement of the plasma tracer concentration for the calculation $25(\mathrm{OH}) \mathrm{D}_{3}$ half-life $[5,10]$.

Dietary nutrient intakes were quantitatively assessed with methods appropriate to each country. In The Gambia, trained field workers weighed all meals, snacks and drinks consumed over 2 days and coding of the dietary records was performed using The Gambian Food Composition Tables [12]. In the UK, a 4-day food diary was completed during one weekend and two week days. All dietary data were entered at MRC HNR using DINO (Diet In Nutrients Out), an in-house analysis program to calculate nutrient intakes [13].

Sample processing and laboratory analysis

Blood was collected into tubes containing either ethylenediaminetetraacetic acid (EDTA) or lithium heparin anticoagulant (S-Monovettes, Sarstedt Ltd, Leicester, UK) and plasma separated by centrifugation at $1800 \mathrm{~g}$ for $20 \mathrm{~min}$ at $4{ }^{\circ} \mathrm{C}$. Samples were stored at $-70{ }^{\circ} \mathrm{C}$. Collected urine was mixed thoroughly and acidified (Hydrochloric acid, $10 \mathrm{~mL} / \mathrm{L}$ Fisher 
Scientific, Loughborough, UK), and aliquots were stored at $-20^{\circ} \mathrm{C}$. Gambian samples were shipped for analysis to MRC HNR, Cambridge, on dry ice.

Plasma calcium, phosphate, creatinine and albumin and urinary calcium $(u \mathrm{Ca})$, urinary phosphate $(u \mathrm{P})$ and urinary creatinine $(u \mathrm{Cr})$ were measured on the Kone Lab $20 \mathrm{i}$ clinical chemistry analyser platform (Kone, Espoo, Finland). Plasma osteocalcin (OC) and bone alkaline phosphatase (BAP) were measured on the DiaSorin Liason platform (DiaSorin Ltd., Dartford, UK). Carboxy-terminal cross-linking telopeptide of type I collagen $(\beta-C T X)$ was measured by ELISA (Immunodiagnostics Systems Ltd., Tyne \& Wear, UK). EDTA plasma was used for analysis of PTH by immunoassay (Immulite, Siemens Healthcare Diagnostics Ltd, Camberley, UK), and C-terminal fibroblast growth factor-23 (FGF23) was analysed using a secondgeneration C-terminal, two-site enzyme-linked immunosorbent assay (Immutopics Inc., San Clemente, CA). Plasma $1,25(\mathrm{OH})_{2} \mathrm{D}$ was measured with a radioimmunoassay (IDS Ltd., Tyne and Wear, UK). DBP was measured by radial immunodiffusion assay with polyclonal antibody [14], and $24,25(\mathrm{OH})_{2} \mathrm{D}$ was analysed by UPLCMS/MS (method modified from [15], both at KU Leuven, Belgium).

Assay performance at MRC HNR was monitored using kit and in-house controls and under strict standardisation according to ISO 9001:2000. Quality assurance of $25(\mathrm{OH}) \mathrm{D}, 1,25(\mathrm{OH})_{2} \mathrm{D}$ and PTH assays was performed as part of the Vitamin D External Quality Assessment Scheme (www. deqas.org) and the National External Quality Assessment Scheme (www.ukneqas.org.uk) and was within accepted limits. In addition, an aliquot of a pooled plasma sample was assayed in each batch to monitor possible drift over time and to provide running quality assurance for analytes where no external reference material was available. Further details of assay performance are described in [16].

\section{Mass spectrometry}

$25(\mathrm{OH}) \mathrm{D}_{2}, 25(\mathrm{OH}) \mathrm{D}_{3}$, and $\mathrm{d}_{3}-25(\mathrm{OH}) \mathrm{D}_{3}$ were measured by UPLC-MS/MS as described previously [10]. Intra- and interassay coefficients of variation were $<10 \%$ for all analytes. Total $25(\mathrm{OH}) \mathrm{D}$ was calculated from the sum of $25(\mathrm{OH}) \mathrm{D}_{2}$ and $25(\mathrm{OH}) \mathrm{D}_{3}$.

\section{Data analysis}

$25(\mathrm{OH}) \mathrm{D}_{3}$ half-life was calculated from the slope of plasma $\mathrm{d}_{3}-25(\mathrm{OH}) \mathrm{D}_{3}$ disappearance using the line of best fit of the natural $\log$ of $\mathrm{d}_{3}-25(\mathrm{OH}) \mathrm{D}_{3}$ concentration against time [10]. One additional Gambian participant was removed from the analysis because $25(\mathrm{OH}) \mathrm{D}_{3}$ half-life values were more than 3 interquartile ranges beyond the 75th centile [10] and had a major impact on relationships between half-life and other variables when analysed together with the remaining participants $(n=36)$. Body mass index was calculated as weight divided by the square of height $\left(\mathrm{kg} / \mathrm{m}^{2}\right)$. Plasma calcium was adjusted for albumin $\left(\mathrm{Ca}_{\mathrm{alb}}\right)$ by normalising to an albumin concentration of $40 \mathrm{~g} / \mathrm{L}$ and a correction factor of $\pm 0.02 \mathrm{mmol}$ calcium/g albumin above or below $36 \mathrm{~g} / \mathrm{L}$ [16]. Fasting 2-h $u \mathrm{Ca}$ and $u \mathrm{P}$ were divided by $u \mathrm{Cr}$ to derive $u \mathrm{Ca} / u \mathrm{Cr}$ and $u \mathrm{P} /$ $u \mathrm{Cr}$ ratios. Statistics were performed in Stata 11 (Stata Corp, TX, USA). Normally distributed data are presented as means and standard deviation (SD). Skewed data were log-transformed and are presented as geometric means and $95 \%$ confidence interval. There were no differences between day 1 and day 21 values of plasma and urinary analytes as tested with paired Student's $t$ tests, so subsequent analysis was performed with the mean values. Unpaired Student's $t$ tests were used to test for differences between populations (Table 1). Statistical analyses were performed on countries separately, due to clustering of data and country-specific associations, and subsequently with data for countries combined with the inclusion of country in the model. In the combined country models, a country $\times$ independent-variable interaction was also tested.

Simple regression was used to evaluate anthropometric, biochemical and dietary predictors of $25(\mathrm{OH}) \mathrm{D}_{3}$ half-life (Table 2) or 25(OH)D plasma concentration (Table 3) for the countries separately and for the countries combined. Multiple linear regression was used to identify variables that independently predicted $25(\mathrm{OH}) \mathrm{D}_{3}$ half-life (Table 4 ) or $25(\mathrm{OH}) \mathrm{D}_{3}$ (Table 5). Throughout, the same full starting models were used with variables for inclusion selected a priori on the basis of a theoretical biological mechanism and/or a significant relationship as assessed with simple regression. These variables were $25(\mathrm{OH}) \mathrm{D}, 1,25(\mathrm{OH})_{2} \mathrm{D}$, DBP, $\mathrm{Ca}_{\mathrm{alb}}$, weight, age, PTH, FGF23, plasma phosphate and dietary calcium intake. Dietary phosphate intake and the plasma concentration of $24,25(\mathrm{OH})_{2} \mathrm{D}$ were not included in multiple regression because of their highly significant and strong correlation with calcium intake (phosphate intake $r=0.87, P<0.0001$ for countries combined) and plasma $25(\mathrm{OH}) \mathrm{D}\left(24,25(\mathrm{OH})_{2} \mathrm{D} r=0.94\right.$, $P<0.0001$ for countries combined), respectively. Variables that were not significant and did not reduce variance were removed, and the final models were chosen based on robustness after the examination of relationships between all variables. Regression coefficients (slope) represent the change in $25(\mathrm{OH}) \mathrm{D}_{3}$ half-life or $25(\mathrm{OH}) \mathrm{D}$ plasma concentration for a 1-unit change in the predictor variable. For log-transformed variables $\left(1,25(\mathrm{OH})_{2} \mathrm{D}, \mathrm{PTH}, \mathrm{FGF} 23\right.$, $u \mathrm{Ca} / u \mathrm{Cr}, u \mathrm{P} / u \mathrm{Cr}$, and plasma $\mathrm{BAP}, \mathrm{OC}$ and $\beta$-CTX), the regression coefficient represents the change in $25(\mathrm{OH}) \mathrm{D}_{3}$ 
Table 1 Participant age, anthropometric data, biochemistry and nutrient intakes (mean (SD)) by country

$\begin{array}{lll}\begin{array}{l}\text { The Gambia } \\ (n=18)\end{array} & \text { UK } & P \text { value }^{\mathrm{a}}\end{array}$

Age and anthropometry

\begin{tabular}{|c|c|c|c|}
\hline Age, years & $29.2(3.2)$ & $29.3(4.4)$ & 1.0 \\
\hline Weight, kg & $64.5(8.3)$ & $73.3(10.9)$ & 0.01 \\
\hline Height, m & $1.74(0.06)$ & $1.80(0.07)$ & 0.009 \\
\hline BMI, $\mathrm{kg} / \mathrm{m}^{2}$ & $21.3(2.0)$ & $22.6(2.3)$ & 0.08 \\
\hline Fat mass, $\mathrm{kg}$ & $7.5(2.6)$ & $10.3(4.3)$ & 0.02 \\
\hline Fat-free mass, $\mathrm{kg}$ & $55.7(6.7)$ & $62.6(8.0)$ & 0.009 \\
\hline \multicolumn{4}{|l|}{ Biochemistry } \\
\hline $25(\mathrm{OH}) \mathrm{D}_{2}, \mathrm{nmol} / \mathrm{L}$ & $0.6(0.2)$ & $2.1(1.0)$ & $<0.0001$ \\
\hline $25(\mathrm{OH}) \mathrm{D}_{3}, \mathrm{nmol} / \mathrm{L}$ & $68.4(13.1)$ & $26.5(10.8)$ & $<0.0001$ \\
\hline $25(\mathrm{OH}) \mathrm{D}, \mathrm{nmol} / \mathrm{L}$ & $69.0(13.2)$ & $28.6(11.0)$ & $<0.0001$ \\
\hline $1,25(\mathrm{OH})_{2} \mathrm{D}, \mathrm{pmol} / \mathrm{L}^{\mathrm{b}}$ & $181(165,197)$ & $120(109,132)$ & $<0.0001$ \\
\hline $24,25(\mathrm{OH})_{2} \mathrm{D}, \mathrm{nmol} / \mathrm{L}$ & $6.5(1.3)$ & $1.9(1.4)$ & $<0.0001$ \\
\hline DBP, mg/L & $259(33)$ & $268(23)$ & 0.4 \\
\hline Albumin, $\mathrm{g} / \mathrm{L}$ & $36.4(2.2)$ & $41.0(2.4)$ & $<0.0001$ \\
\hline $\mathrm{Ca}_{\mathrm{alb}}, \mathrm{mmol} / \mathrm{L}$ & $2.25(0.06)$ & $2.27(0.06)$ & 0.2 \\
\hline Phosphate, $\mathrm{mmol} / \mathrm{L}$ & $1.04(0.14)$ & $1.05(0.10)$ & 0.9 \\
\hline $\mathrm{PTH}, \mathrm{ng} / \mathrm{L}^{\mathrm{b}}$ & $50.1(41.9,60.0)$ & $32.8(27.4,39.3)$ & $<0.01$ \\
\hline FGF23, RU/mL ${ }^{\mathrm{b}}$ & $79.2(58.6,106.9)$ & $57.0(52.5,62.0)$ & 0.03 \\
\hline Plasma BAP, $\mu \mathrm{g} / \mathrm{L}^{\mathrm{b}}$ & $17.3(12.8,23.3)$ & $11.8(10.1,13.9)$ & 0.02 \\
\hline Plasma OC, $\mu \mathrm{g} / \mathrm{L}^{\mathrm{b}}$ & $15.0(12.7,17.6)$ & $10.8(9.3,12.6)$ & 0.004 \\
\hline Plasma $\beta-C T X, \mu g / L^{b}$ & $1.0(0.9,1.2)$ & $0.6(0.5,0.7)$ & $<0.0001$ \\
\hline$u \mathrm{Ca} / u \mathrm{Cr}, \mathrm{mmol} / \mathrm{mmol}^{\mathrm{b}}$ & $0.06(0.04,0.09)$ & $0.17(0.13,0.26)$ & $<0.0001$ \\
\hline$u \mathrm{P} / u \mathrm{Cr}, \mathrm{mmol} / \mathrm{mmol}^{\mathrm{b}}$ & $0.73(0.59,0.90)$ & $0.99(0.83,1.17)$ & 0.02 \\
\hline \multicolumn{4}{|l|}{ Nutrient intake ${ }^{\mathrm{c}}$} \\
\hline Energy, kJ/day & 8267 (2383) & 9804 (1931) & 0.04 \\
\hline Fat, g/day & $42(17)$ & $94(27)$ & $<0.001$ \\
\hline Protein, g/day & $68(26)$ & $90(19)$ & 0.005 \\
\hline Carbohydrate, g/day & $358(115)$ & $281(60)$ & 0.02 \\
\hline Calcium, mg/day & $358(177)$ & $1087(248)$ & $<0.0001$ \\
\hline Phosphate, mg/day & $803(332)$ & $1603(300)$ & $<0.0001$ \\
\hline Magnesium, mg/day & $506(222)$ & $361(94)$ & 0.02 \\
\hline
\end{tabular}

$D B P$ vitamin $\mathrm{D}$ binding protein, $C a_{\text {alb }}$ plasma calcium corrected for albumin, $P T H$ parathyroid hormone, FGF23 fibroblast growth factor 23, $B A P$ bone alkaline phosphatase, $O C$ osteocalcin, $\beta$-CTX $\beta$-carboxyterminal collagen crosslinks, $u \mathrm{Ca} / u \mathrm{Cr}$ creatinine corrected fasting $2-\mathrm{h}$ urinary $\mathrm{Ca}, u \mathrm{P} / u \mathrm{Cr}$ creatinine corrected fasting 2-h urinary phosphate ${ }^{a}$ Country differences as tested by unpaired, two-tailed Student's $t$ test

${ }^{\mathrm{b}}$ Data are presented as mean (SD) or geometric mean $(95 \% \mathrm{CI})$ of samples collected on day 1 and day 21

${ }^{\mathrm{c}}$ Daily nutrient intakes were assessed by 2-day weighed dietary assessment in The Gambia and 4-day food diary in the UK

half-life or $25(\mathrm{OH}) \mathrm{D}$ plasma concentration for a $100 \%$ change in the predictor variable. Relationships were considered significant where $P<0.05$, and a trend for a relationship was noted where $P \geq 0.05$ and $P<0.1$.

\section{Results}

\section{Country comparisons}

Anthropometric, biochemical and dietary data are shown in Table 1. Gambian participants were significantly shorter and lighter than UK participants and had lower fat mass and fatfree mass. Plasma $25(\mathrm{OH}) \mathrm{D}, 1,25(\mathrm{OH})_{2} \mathrm{D}, 24,25(\mathrm{OH})_{2} \mathrm{D}$, PTH and FGF23 concentrations were higher and plasma DBP, $\mathrm{Ca}_{\text {alb }}$ and plasma phosphate were not different in Gambian compared to UK participants. Markers of bone resorption and formation were also higher in Gambian participants. Gambian macro- and micronutrient intakes were generally lower than those in the UK with the exception of carbohydrate and magnesium which were significantly higher. Consistent with the lower dietary calcium and phosphate intakes, fasting 2-h urinary excretions in The Gambia were lower than those in the UK. Mean $25(\mathrm{OH}) \mathrm{D}_{3}$ half-life was 14.7 (3.5) days and 15.6 (2.5)days in The Gambia and UK, respectively, and was not significantly different between countries (difference 0.9 days; $P=0.2$ ) [10].

\section{Predictors of $25(\mathrm{OH}) \mathrm{D}_{3}$ half-life}

In Gambian participants, $25(\mathrm{OH}) \mathrm{D}_{3}$ half-life was negatively associated with $\mathrm{Ca}_{\text {alb }}$ and positively associated with age and DBP (Table 2) in simple regression models. There were trends towards a negative association with PTH $(P=0.09)$ and positive with $u \mathrm{P} / u \mathrm{Cr}(P=0.08)$. In contrast, in the UK participants in simple regression models, none of the measured variables were significantly associated with $25(\mathrm{OH}) \mathrm{D}_{3}$ half-life. With the countries combined, where the country was also included in the model, $\mathrm{Ca}_{\mathrm{alb}}$ was negatively associated and age, DBP and plasma phosphate were positively associated with $25(\mathrm{OH}) \mathrm{D}_{3}$ half-life. Near-significant relationships were also observed with PTH (inversely) and $u \mathrm{P} / u \mathrm{Cr}$ (a positive association); in none of these models was country a significant predictor of $25(\mathrm{OH}) \mathrm{D}_{3}$ half-life. However, the pattern of the relationship between half-life and both $\mathrm{Ca}_{\mathrm{alb}}$ and age differed between countries. There were significant country $\times$ age $(P=0.03)$ and country $\times \mathrm{Ca}_{\text {alb }}$ interactions $(P=0.006)$ such that the positive association with age and negative association with $\mathrm{Ca}_{\text {alb }}$ was of a greater magnitude in the Gambians and not significant in the UK participants.

Results of the multivariate model (Table 3) were partly consistent with simple regression analysis. $\mathrm{Ca}_{\text {alb }}$ and $1,25(\mathrm{OH})_{2} \mathrm{D}$ negatively predicted and $25(\mathrm{OH}) \mathrm{D}$ and $\mathrm{DBP}$ positively predicted $25(\mathrm{OH}) \mathrm{D}_{3}$ half-life in Gambians. Together, these variables explained $79 \%$ (adjusted $R^{2}$ ) of the variation in $25(\mathrm{OH}) \mathrm{D}_{3}$ half-life. There were no significant associations in UK participants (Table 3). In the model for the 
Table 2 Regression analysis of $25(\mathrm{OH}) \mathrm{D}_{3}$ half-life and other variables by country and for the countries combined

\begin{tabular}{|c|c|c|c|c|c|c|}
\hline & \multicolumn{2}{|l|}{ The Gambia } & \multicolumn{2}{|l|}{ UK } & \multicolumn{2}{|c|}{ Countries combined and corrected for country } \\
\hline & Slope $(95 \%$ CI $)$ & $P$ value & Slope $(95 \%$ CI) & $P$ value & Slope $(95 \%$ CI $)$ & $P$ value \\
\hline \multicolumn{7}{|l|}{ Age and anthropometry } \\
\hline Age, years & $0.63(0.16,1.10)$ & 0.01 & $0.07(-0.23,0.37)$ & 0.6 & $0.27(0.002,0.54)$ & $0.05^{b}$ \\
\hline Weight, kg & $0.09(-0.13,0.31)$ & 0.4 & $-0.02(-0.14,0.10)$ & 0.7 & $0.02(-0.09,0.13)$ & 0.7 \\
\hline BMI, $\mathrm{kg} / \mathrm{m}^{2}$ & $0.34(-0.61,1.28)$ & 0.5 & $-0.19(-0.75,0.37)$ & 0.5 & $0.03(-0.48,0.54)$ & 0.9 \\
\hline Fat mass, $\mathrm{kg}$ & $0.14(-0.56,0.86)$ & 0.7 & $-0.02(-0.33,0.28)$ & 0.9 & $0.02(-0.28,0.33)$ & 0.9 \\
\hline Fat-free mass, $\mathrm{kg}$ & $0.12(-0.15,0.39)$ & 0.4 & $-0.03(-0.19,0.14)$ & 0.7 & $0.04(-0.11,0.18)$ & 0.6 \\
\hline \multicolumn{7}{|c|}{ Markers of vitamin D, Ca and P metabolism } \\
\hline $25(\mathrm{OH}) \mathrm{D}, \mathrm{nmol} / \mathrm{L}$ & $0.09(-0.04,0.22)$ & 0.2 & $-0.02(-0.14,0.10)$ & 0.7 & $0.04(-0.04,0.13)$ & 0.3 \\
\hline $1,25(\mathrm{OH})_{2} \mathrm{D}, \mathrm{pmol} / \mathrm{L}^{\mathrm{c}}$ & $4.70(-5.49,14.88)$ & 0.3 & $-1.67(-8.48,5.12)$ & 0.6 & $1.27(-4.54,7.08)$ & 0.7 \\
\hline $24,25(\mathrm{OH})_{2} \mathrm{D}, \mathrm{nmol} / \mathrm{L}$ & $0.64(-0.72,2.01)$ & 0.3 & $-0.09(-1.04,0.86)$ & 0.8 & $-0.26(-0.53,1.05)$ & 0.5 \\
\hline $\mathrm{DBP}, \mathrm{mg} / \mathrm{L}$ & $0.06(0.14,0.11)$ & 0.01 & $0.004(-0.05,0.06)$ & 0.9 & $0.04(0.007,0.08)$ & 0.02 \\
\hline Albumin, $\mathrm{g} / \mathrm{L}$ & $-0.18(-1.03,0.67)$ & 0.7 & $0.25(-0.28,0.78)$ & 0.3 & $0.06(-0.41,0.53)$ & 0.8 \\
\hline $\mathrm{Ca}_{\mathrm{alb}}, \mathrm{mmol} / \mathrm{L}$ & $-44.6(-65.5,-23.6)$ & $<0.0001$ & $-1.31(-23.6,21.0)$ & 0.9 & $-23.0(-39.3,-6.58)$ & $0.007^{b}$ \\
\hline Phosphate, $\mathrm{mmol} / \mathrm{L}$ & $8.37(-4.07,20.8)$ & 0.2 & $9.20(-2.85,21.26)$ & 0.1 & $8.66(0.43,16.8)$ & 0.04 \\
\hline $\mathrm{PTH}, \mathrm{ng} / \mathrm{L}^{\mathrm{c}}$ & $-4.06(-8.87,0.71)$ & 0.09 & $-1.42(-5.02,2.18)$ & 0.4 & $-2.73(-5.59,0.13)$ & 0.06 \\
\hline FGF23, RU/mL ${ }^{\mathrm{c}}$ & $0.19(-2.92,3.31)$ & 0.9 & $-0.71(-8.66,7.24)$ & 0.9 & $0.13(-2.32,2.59)$ & 0.9 \\
\hline$u \mathrm{Ca} / u \mathrm{Cr}, \mathrm{mmol} / \mathrm{mmol}^{\mathrm{c}}$ & $-0.78(-2.97,1.40)$ & 0.5 & $-1.21(-3.38,0.96)$ & 0.3 & $-0.92(-2.38,0.53)$ & 0.2 \\
\hline$u \mathrm{P} / u \mathrm{Cr}, \mathrm{mmol} / \mathrm{mmol}^{\mathrm{c}}$ & $3.46(-0.51,7.42)$ & 0.08 & $1.33(-2.50,5.16)$ & 0.5 & $2.64(-0.006,5.28)$ & 0.05 \\
\hline \multicolumn{7}{|c|}{ Markers of bone metabolism } \\
\hline Plasma BAP, $\mu \mathrm{g} / \mathrm{L}^{\mathrm{c}}$ & $-0.94(-4.05,2.17)$ & 0.5 & $1.10(-3.01,5.22)$ & 0.6 & $-0.49(-2.75,1.78)$ & 0.7 \\
\hline Plasma OC, $\mu \mathrm{g} / \mathrm{L}^{\mathrm{c}}$ & $-0.26(-6.07,5.54)$ & 0.9 & $-0.49(-4.83,3.85)$ & 0.8 & $-0.37(-3.82,3.09)$ & 0.8 \\
\hline Plasma $\beta-\mathrm{CTX}, \mu \mathrm{g} / \mathrm{L}^{\mathrm{c}}$ & $-2.02(-8.30,4.27)$ & 0.5 & $0.47(-3.57,4.50)$ & 0.8 & $-0.64(-4.12,2.83)$ & 0.7 \\
\hline \multicolumn{7}{|l|}{ Nutrient intake } \\
\hline Calcium intake, mg/day & $0.003(-0.007,0.14)$ & 0.4 & $0.003(-0.002,0.008)$ & 0.2 & $0.004(-0.001,0.008)$ & 0.1 \\
\hline Phosphate intake, mg/day & $0.003(-0.002,0.01)$ & 0.2 & $0.00003(-0.004,0.004)$ & 1.0 & $0.002(-0.002,0.005)$ & 0.3 \\
\hline
\end{tabular}

Italicized values show significant $(P<0.05)$ associations with $25(\mathrm{OH}) \mathrm{D}_{3}$ half-life or a trend for an association $(P<0.1, \geq 0.5)$

${ }^{\text {a }}$ Country was a not significant predictor for any variable

${ }^{\mathrm{b}}$ There were significant country $\times$ age $(P=0.03)$ and country $\times \mathrm{Ca}_{\mathrm{alb}}(P=0.006)$ interactions

${ }^{\mathrm{c}}$ Log-transformed variables. Coefficients represent change in $25(\mathrm{OH}) \mathrm{D}_{3}$ half-life for $100 \%$ change in the predictor variable

Table 3 Predictors of $25(\mathrm{OH}) \mathrm{D}_{3}$ half-life in Gambian and UK participants and for countries combined in multivariate regression model

\begin{tabular}{|c|c|c|c|c|c|c|}
\hline & \multicolumn{2}{|l|}{ Gambia $(n=18)$} & \multicolumn{2}{|l|}{$\mathrm{UK}(n=18)$} & \multicolumn{2}{|c|}{ Countries combined $(n=36)$} \\
\hline & Slope $(95 \%$ CI $)$ & $P$ value & Slope $(95 \%$ CI $)$ & $P$ value & Slope $(95 \%$ CI) & $P$ value \\
\hline $25(\mathrm{OH}) \mathrm{D}$ & $0.12(0.04,0.19)$ & 0.004 & $-0.02(-0.18,0.14)$ & 0.8 & & \\
\hline $1,25(\mathrm{OH})_{2} \mathrm{D}^{\mathrm{a}}$ & $-6.33(-12.1,-0.54)$ & 0.04 & $-1.3(-9.6,7.0)$ & 0.7 & & \\
\hline DBP & $0.04(0.01,0.07)$ & 0.009 & $-0.003(-0.07,0.07)$ & 0.9 & & \\
\hline $\mathrm{Ca}_{\mathrm{alb}}$ & $-44.5(-60.7,-28.2)$ & $<0.0001$ & $-2.56(-30.5,25.4)$ & 0.8 & $-24.2(-38.3,-10.0)$ & 0.001 \\
\hline $\mathrm{PTH}^{\mathrm{a}}$ & & & & & $-3.20(-5.53,-0.86)$ & 0.009 \\
\hline Plasma phosphate & & & & & $8.38(1.62,15.14)$ & 0.02 \\
\hline Country & & & & & & 0.9 \\
\hline
\end{tabular}

${ }^{a}$ Log-transformed variables. Coefficients represent change in $25(\mathrm{OH}) \mathrm{D}_{3}$ half-life for $100 \%$ change in the predictor variable 
Table 4 Regression analysis of plasma 25(OH)D and other variables by country and for the countries combined

\begin{tabular}{|c|c|c|c|c|c|c|}
\hline & \multicolumn{2}{|l|}{ The Gambia } & \multicolumn{2}{|l|}{ UK } & \multicolumn{2}{|c|}{ Countries combined and corrected for country } \\
\hline & Slope $(95 \%$ CI $)$ & $P$ value & Slope $(95 \% \mathrm{CI})$ & $P$ value & Slope $(95 \%$ CI $)$ & $P$ value \\
\hline \multicolumn{7}{|l|}{ Age and anthropometry } \\
\hline Age, years & $-0.17(-2.33,1.99)$ & 0.9 & $0.71(-0.57,1.99)$ & 0.3 & $0.40(-0.71,1.51)$ & 0.5 \\
\hline Weight, kg & $0.53(-0.27,1.32)$ & 0.2 & $0.25(-0.27,0.76)$ & 0.3 & $0.35(-0.76,0.77)$ & 0.1 \\
\hline BMI, $\mathrm{kg} / \mathrm{m}^{2}$ & $1.83(-1.63,5.29)$ & 0.3 & $0.74(-1.73,3.22)$ & 0.5 & $1.19(-0.77,3.16)$ & 0.2 \\
\hline Fat mass, $\mathrm{kg}$ & $0.31(-2.35,2.98)$ & 0.8 & $0.72(-0.58,2.01)$ & 0.3 & $0.61(-0.58,1.79)$ & 0.3 \\
\hline Fat-free mass, kg & $0.72(-0.25,1.69)$ & 0.1 & $0.25(-0.47,0.96)$ & 0.5 & $0.44(-0.12,1.00)$ & 0.1 \\
\hline \multicolumn{7}{|c|}{ Markers of vitamin D, Ca and P metabolism } \\
\hline $25(\mathrm{OH}) \mathrm{D}, \mathrm{nmol} / \mathrm{L}$ & - & - & - & - & - & - \\
\hline $1,25(\mathrm{OH})_{2} \mathrm{D}, \mathrm{pmol} / \mathrm{L}^{\mathrm{b}}$ & $29.8(-5.97,65.6)$ & 0.1 & $20.9(-6.98,48.8)$ & 0.1 & $25.0(3.72,46.3)$ & 0.02 \\
\hline $24,25(\mathrm{OH})_{2} \mathrm{D}, \mathrm{nmol} / \mathrm{L}$ & $6.26(2.20,10.3)$ & 0.005 & $6.94(5.02,8.87)$ & $<0.0001$ & $6.62(4.52,8.72)$ & $<0.0001$ \\
\hline $\mathrm{DBP}, \mathrm{mg} / \mathrm{L}$ & $0.04(-0.16,0.25)$ & 0.7 & $-0.11(-0.35,0.13)$ & 0.4 & $-0.006(-0.16,0.14)$ & 0.9 \\
\hline Albumin, $\mathrm{g} / \mathrm{L}$ & $-3.30(-5.96,-0.63)$ & 0.02 & $0.51(-1.88,2.90)$ & 0.7 & $-1.22(-3.03,0.59)$ & $0.2^{\mathrm{c}}$ \\
\hline $\mathrm{Ca}_{\mathrm{alb}}, \mathrm{mmol} / \mathrm{L}$ & $2.46(-115,120)$ & 1.0 & $-45.1(-139.8,49.7)$ & 0.3 & $-21.3(-93.2,50.7)$ & 0.6 \\
\hline Plasma phosphate, $\mathrm{mmol} / \mathrm{L}$ & $12.5(-36.3,61.4)$ & 0.6 & $-11.5(-68.1,45.0)$ & 0.7 & $4.38(-30.3,39.1)$ & 0.8 \\
\hline PTH, ng/L ${ }^{b}$ & $-8.62(-27.6,10.4)$ & 0.4 & $-11.4(-26.3,3.44)$ & 0.1 & $-10.0(-21.4,1.36)$ & 0.08 \\
\hline FGF23, RU/mL ${ }^{\mathrm{b}}$ & $-5.34(16.6,5.94)$ & 0.3 & $-6.98(-41.6,27.6)$ & 0.7 & $-5.46(-15.0,4.08)$ & 0.3 \\
\hline$u \mathrm{Ca} / u \mathrm{Cr}, \mathrm{mmol} / \mathrm{mmol}^{\mathrm{b}}$ & $-1.61(-9.86,6.64)$ & 0.7 & $-3.81(-13.5,5.87)$ & 0.4 & $-2.32(-8.18,3.53)$ & 0.4 \\
\hline$u \mathrm{P} / u \mathrm{Cr}, \mathrm{mmol} / \mathrm{mmol}^{\mathrm{b}}$ & $-0.55(-16.9,15.8)$ & 0.9 & $-13.6(-29.0,1.78)$ & 0.08 & $-5.61(-16.5,5.30)$ & 0.3 \\
\hline \multicolumn{7}{|c|}{ Markers of bone metabolism } \\
\hline Plasma BAP, $\mu \mathrm{g} / \mathrm{L}^{\mathrm{b}}$ & $3.90(-7.66,15.5)$ & 0.5 & $7.38(-10.4,25.1)$ & 0.4 & $4.68(-4.16,13.5)$ & 0.3 \\
\hline Plasma OC, $\mu \mathrm{g} / \mathrm{L}^{\mathrm{b}}$ & $-10.3(-31.3,10.7)$ & 0.3 & $3.13(-15.8,22.1)$ & 0.7 & $-3.98(-17.6,9.61)$ & 0.6 \\
\hline Plasma $\beta-\mathrm{CTX}, \mu \mathrm{g} / \mathrm{L}^{\mathrm{b}}$ & $5.20(18.4,28.8)$ & 0.6 & $-1.82(-19.5,15.8)$ & 0.8 & $1.31(-12.5,15.1)$ & 0.8 \\
\hline \multicolumn{7}{|l|}{ Nutrient intake } \\
\hline Calcium intake, mg/day & $-0.007(-0.05,0.03)$ & 0.7 & $0.0007(-0.02,0.02)$ & 0.9 & $-0.002(-0.02,0.02)$ & 0.9 \\
\hline Phosphate intake, mg/day & $0.01(-0.009,0.03)$ & 0.2 & $0.002(-0.02,0.02)$ & 0.8 & $0.007(-0.006,0.02)$ & 0.3 \\
\hline
\end{tabular}

Italicized values show significant $(P<0.05)$ associations with $25(\mathrm{OH}) \mathrm{D}_{3}$ or a trend for an association $(P<0.1, \geq 0.5)$

${ }^{a}$ Country was a significant predictor for all variables $(P<0.0001)$ with the exception of $24,25(\mathrm{OH})_{2} \mathrm{D}$

${ }^{\mathrm{b}}$ Log-transformed variables. Coefficients represent change in $25(\mathrm{OH}) \mathrm{D}$ plasma concentration for $100 \%$ change in the predictor variable

${ }^{\mathrm{c}}$ There was a significant country $\times$ albumin interaction $(P=0.03)$

countries combined, $\mathrm{Ca}_{\mathrm{alb}}$ and PTH were negatively associated and plasma phosphate positively associated with half-life (Table 3). Country was nonsignificant.
In simple and multivariate regression, we obtained similar results if $\mathrm{pH}$-adjusted ionised calcium or plasma calcium (independent of plasma albumin) were used instead of $\mathrm{Ca}_{\mathrm{alb}}$

Table 5 Predictors of plasma 25(OH)D in Gambian and UK participants and for countries combined in multivariate regression model

\begin{tabular}{|c|c|c|c|c|c|c|}
\hline & \multicolumn{2}{|l|}{ Gambia $(n=18)$} & \multicolumn{2}{|l|}{$\mathrm{UK}(n=18)$} & \multicolumn{2}{|c|}{ Countries combined $(n=36)$} \\
\hline & Slope $(95 \%$ CI $)$ & $P$ value & Slope $(95 \%$ CI) & $P$ value & Slope $(95 \% \mathrm{CI})$ & $P$ value \\
\hline $1,25(\mathrm{OH})_{2} \mathrm{D}^{\mathrm{a}}$ & $33.2(0.13,66.3)$ & 0.05 & $21.3(-3.50,46.2)$ & 0.09 & $29.3(9.89,48.8)$ & 0.004 \\
\hline $\mathrm{Ca}_{\mathrm{alb}}$ & & & $-68.3(-152,15.8)$ & 0.1 & & \\
\hline $\mathrm{PTH}^{\mathrm{a}}$ & $-15.7(33.8,2.4)$ & 0.09 & $-14.6(-28.4,-0.76)$ & 0.04 & $-9.76(-19.6,0.12)$ & 0.05 \\
\hline 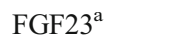 & $-10.5(-21.4,0.3)$ & 0.06 & & & & \\
\hline Weight & & & & & $0.45(0.08,0.82)$ & 0.02 \\
\hline Country & & & & & & $<0.0001$ \\
\hline
\end{tabular}

${ }^{\mathrm{a}}$ Log-transformed variables. Coefficients represent change in $25(\mathrm{OH}) \mathrm{D}$ plasma concentration for $100 \%$ change in predictor variable 
for countries analysed separately and combined (data not shown).

\section{Predictors of $25(\mathrm{OH}) \mathrm{D}$}

There were few significant associations with $25(\mathrm{OH}) \mathrm{D}$ in simple regression models: $24,25(\mathrm{OH})_{2} \mathrm{D}$ was positively associated with $25(\mathrm{OH}) \mathrm{D}$ in both countries and for the countries combined. Plasma albumin inversely predicted $25(\mathrm{OH}) \mathrm{D}$ in Gambians, and there were trends for a positive association with $1,25(\mathrm{OH})_{2} \mathrm{D}(P=0.097)$ in Gambians and a negative association with $u \mathrm{P} / u \mathrm{Cr}(P=0.08)$ in $\mathrm{UK}$ participants (Table 4$)$. When the countries were combined, there was a positive association with $1,25(\mathrm{OH})_{2} \mathrm{D}$ and a trend towards a negative association with PTH $(P=0.08)$ (Table 4); country was a highly significant predictor of $25(\mathrm{OH}) \mathrm{D}$ (The Gambia $>\mathrm{UK}, P<0.0001)$ in all models with the exception of $24,25(\mathrm{OH})_{2} \mathrm{D}$. There was a significant country $\times$ albumin interaction $(P=0.03)$, such that there was a strong negative association with albumin in the Gambians and a nonsignificant positive association in the UK participants.

In country-specific multivariate models, $1,25(\mathrm{OH}) \mathrm{D}_{2}$ positively predicted $25(\mathrm{OH}) \mathrm{D}$, and there was a trend for inverse associations with FGF23 and PTH for Gambians (Table 5). In the UK, there was a significant inverse association with $\mathrm{PTH}$, together with near-significant negative and positive associations with $\mathrm{Ca}_{\text {alb }}$ and $1,25(\mathrm{OH})_{2} \mathrm{D}$, respectively (Table 5). When the countries were combined, country was the strongest predictor of $25(\mathrm{OH}) \mathrm{D}$, accounting for $74 \%$ (adjusted $R^{2}$ ) of the variation in $25(\mathrm{OH}) \mathrm{D}$. However, PTH negatively predicted and $1,25(\mathrm{OH})_{2} \mathrm{D}$ and weight positively predicted $25(\mathrm{OH}) \mathrm{D}$ and, together with country, accounted for $81 \%$ of the variation (Table 5). There were no significant interactions with country.

\section{Predictors of $24,25(\mathrm{OH})_{2} \mathrm{D}$}

The only consistently significant predictor of $24,25(\mathrm{OH})_{2} \mathrm{D}$ was $25(\mathrm{OH}) \mathrm{D}$, and this was true when data for the countries were combined (with country as a covariate) (coefficient, $0.08 \mathrm{nmol} / \mathrm{L}$ per $1 \mathrm{nmol} / \mathrm{L}$ change in $25(\mathrm{OH}) \mathrm{D} ; P<0.0001)$ and when analysed separately (The Gambia (coefficient, $0.06 \mathrm{nmol} / \mathrm{L} ; P=0.005$ ); the UK (coefficient, $0.11 \mathrm{nmol} / \mathrm{L}$; $P<0.0001))$. PTH also predicted $24,25(\mathrm{OH})_{2} \mathrm{D}$ in the countries combined and The Gambia (a $10 \%$ change in PTH predicts a 0.1 and $0.2 \mathrm{nmol} / \mathrm{L}(P=0.03)$ decrease in $24,25(\mathrm{OH})_{2} \mathrm{D}$, respectively). In multivariate models, there were no predictors of $24,25(\mathrm{OH})_{2} \mathrm{D}$ other than $25(\mathrm{OH}) \mathrm{D}$. There were no associations with FGF23. In combined country models, there were no country interactions.

\section{Discussion}

$25(\mathrm{OH}) \mathrm{D}$ plasma concentration is a biomarker of vitamin $\mathrm{D}$ status and reflects the balance between vitamin D supply from cutaneous synthesis and diet, hepatic $25(\mathrm{OH}) \mathrm{D}$ production and $25(\mathrm{OH}) \mathrm{D}$ expenditure, which represents primarily hydroxylation into $1,25(\mathrm{OH})_{2} \mathrm{D}$ and $24,25(\mathrm{OH})_{2} \mathrm{D}$. Plasma $25(\mathrm{OH}) \mathrm{D}$ may also be influenced by the distribution volume, storage and release of vitamin $\mathrm{D}$ and $25(\mathrm{OH}) \mathrm{D}$ from fat or muscle [17]. Some of the mechanisms controlling 25(OH)D status (whether production or utilisation) and their relative importance in healthy people are not fully elucidated. The measurement of $25(\mathrm{OH}) \mathrm{D}$ half-life provides a measure of $25(\mathrm{OH}) \mathrm{D}$ expenditure that allows investigation of factors that influence plasma $25(\mathrm{OH}) \mathrm{D}$ concentration without the confounding contribution of newly synthesised $25(\mathrm{OH}) \mathrm{D}$. We have investigated the predictors of $25(\mathrm{OH}) \mathrm{D}_{3}$ half-life and of plasma $25(\mathrm{OH}) \mathrm{D}$ in two distinct populations. In the Gambia and when data for the countries were combined, we found that the strongest and most consistent predictor of $25(\mathrm{OH}) \mathrm{D}_{3}$ halflife was $\mathrm{Ca}_{\mathrm{alb}}$ inversely, but PTH inversely and total $25(\mathrm{OH}) \mathrm{D}, 1,25(\mathrm{OH})_{2} \mathrm{D}$, DBP and $p \mathrm{P}$ plasma phosphate positively predicted half-life in multivariate models. However, predictors were different between countries and in models for the countries combined. For total $25(\mathrm{OH}) \mathrm{D}$, PTH was a negative predictor, and $1,25(\mathrm{OH})_{2} \mathrm{D}$ and weight were positive predictors, although country was the major influencing factor. As with half-life, the predictors of plasma $25(\mathrm{OH}) \mathrm{D}$ differed between countries. These data suggest that $25(\mathrm{OH}) \mathrm{D}$ half-life is primarily predicted by factors associated with $25(\mathrm{OH}) \mathrm{D}$ metabolism, whereas $25(\mathrm{OH}) \mathrm{D}$ is most strongly predicted by country, probably as a proxy measure of vitamin D supply from UVB exposure.

Plasma 25(OH)D half-life is likely to be determined by the metabolism of $25(\mathrm{OH}) \mathrm{D}$ to downstream metabolites and by its catabolism, driven primarily by the activities of the enzymes CYP27B1 (producing 1,25(OH) 2 D) and CYP24A1 (catabolising $25(\mathrm{OH}) \mathrm{D}$ and further downstream metabolites) [6]. The activities of these enzymes are stimulated by a large number of factors, the most important being increases in PTH and $1,25(\mathrm{OH})_{2} \mathrm{D}$ and/or low plasma $\mathrm{P}$. We observed associations between these factors and $25(\mathrm{OH}) \mathrm{D}_{3}$ half-life, although these were not consistent between countries, possibly due to genetic or environmental, especially dietary, differences. Low dietary calcium intake [7] and low calcium bioavailability, due to a high-fibre diet [9] or intestinal malabsorption [8, 18, 19], were reported to be associated with a shorter half-life. This is likely to be mediated through the PTH-CYP27B1-CYP24A1 axis, increasing the production of both $1,25(\mathrm{OH})_{2} \mathrm{D}$ and $24,25(\mathrm{OH})_{2} \mathrm{D}[7,20]$. In our study, dietary calcium intake was 3-fold lower in Gambian compared to UK participants, yet contrary to our expectations, there was no difference in mean $25(\mathrm{OH}) \mathrm{D}_{3}$ half-life between the populations [10] and no 
association between half-life and calcium intake. This relationship may be difficult to prove in human studies over the habitual range of calcium intake, particularly in populations where there are other factors that may influence half-life and may require a large sample size. In addition, we may not have observed a difference in half-life between countries because of population differences due to long-term adaptation of vitamin $\mathrm{D}$ metabolism to either low vitamin D supply/high calcium intake (UK) or high vitamin D supply/low calcium intake (The Gambia). This may be due to genetic differences between populations, for example in DBP, vitamin D receptor or hydroxylase enzymes, or the activity of the hydroxylase enzymes regulating $25(\mathrm{OH}) \mathrm{D}$ synthesis and catabolism into $1,25(\mathrm{OH})_{2} \mathrm{D}$ and $24,25(\mathrm{OH})_{2} \mathrm{D}$ and further downstream metabolites. This could be investigated in future studies by the assessment of downstream metabolites of the tracer compound.

In this study, the strongest and most robust relationship with half-life was observed with plasma $\mathrm{Ca}_{\mathrm{alb}}$ although this relationship was not observed in the UK participants when analysed separately. This association, which was not a reflection of differences in plasma albumin concentration, is surprising given that plasma calcium concentration is tightly regulated and thus half-life is more likely to be strongly associated with its regulators, PTH and $1,25(\mathrm{OH})_{2} \mathrm{D}$. In addition, any relationship between plasma calcium and half-life would have been expected, on a theoretic basis, to be positive, with lower plasma calcium resulting in greater usage of 25(OH)D through the PTH-CYP27B1-CYP24A1 axis. The importance of $1,25(\mathrm{OH})_{2} \mathrm{D}$ and PTH in the control of vitamin $\mathrm{D}$ metabolism was shown previously in clinical and animal studies. An increase in plasma $1,25(\mathrm{OH})_{2} \mathrm{D}$ and hyperparathyroidism have been associated with an increase in $25(\mathrm{OH}) \mathrm{D}$ expenditure, whereas hypoparathyroidism was associated with a longer half-life [6]. Consistent with these results, we found a negative relationship between PTH and 25(OH)D half-life. A negative relationship between $1,25(\mathrm{OH})_{2} \mathrm{D}$ and $25(\mathrm{OH}) \mathrm{D}_{3}$ half-life was only observed in Gambians, possibly because of the higher $1,25(\mathrm{OH})_{2} \mathrm{D}$ concentration in this population, likely related to their very low calcium intake.

The mechanisms of the reciprocal regulation of vitamin D and phosphate metabolism are less well established than for calcium [21]. Phosphate deficiency may stimulate CYP27B1, leading to increased plasma $1,25(\mathrm{OH})_{2} \mathrm{D}$ [22], and high phosphate intake or plasma phosphate concentration is associated with an elevated FGF23, which stimulates urinary phosphate excretion and CYP24A1 activity and downregulates CYP27B1. We found a positive relationship between plasma phosphate, $u \mathrm{P} / u \mathrm{Cr}$ and $25(\mathrm{OH}) \mathrm{D}_{3}$ half-life, supporting a role for phosphate in vitamin D metabolism. However, we did not observe a relationship with c-terminal FGF23 or with dietary phosphate intake. This lack of association was also observed in a study in Gambian children where no relationship was found between 25(OH)D half-life and active (intact) FGF23 [23]. We found that FGF23 was elevated in Gambians compared to UK subjects. This may be due to low calcium intake and/or high dietary phosphate/calcium ratio driving elevated $1,25(\mathrm{OH})_{2} \mathrm{D}$. FGF23 is thought to regulate vitamin D metabolism in conjunction with PTH; FGF23 has been shown to increase vitamin $\mathrm{D}$ receptor (VDR), calcium sensing receptor and CYP24A1 expression and inhibit CYP27B1 expression through which it suppresses plasma PTH and potentially influences 25(OH)D half-life [24].

DBP concentration was positively associated with $25(\mathrm{OH}) \mathrm{D}_{3}$ half-life. DBP concentration and genotype may affect vitamin D metabolism and function [25]. Vitamin D metabolites are maintained in the circulation by their binding to DBP. Higher circulating levels of DBP may reduce the fraction of free-25(OH)D, and a longer half-life may be expected. Further, these relationships may be affected by DBP genotype that may [25] or may not [26] alter affinity. However, we have shown earlier that the relationships between free-25(OH)D and half-life are not different to those with 25(OH)D [10].

We also observed a positive association between $25(\mathrm{OH}) \mathrm{D}$ and $25(\mathrm{OH}) \mathrm{D}_{3}$ half-life in Gambian participants. Early observations suggested an influence of $25(\mathrm{OH}) \mathrm{D}$ plasma status on $25(\mathrm{OH}) \mathrm{D}$ half-life, with more rapid turnover in vitamin $\mathrm{D}$ deplete subjects [27], but other studies have failed to reproduce these findings in normal and diseased subjects $[8,18]$. Our results suggest, in Gambian participants at least, that a higher plasma $25(\mathrm{OH}) \mathrm{D}$ concentration is associated with a longer half-life, or that a longer half-life results in a higher $25(\mathrm{OH}) \mathrm{D}$ concentration. We found no relationships between any of the anthropometric measures of size or fatness with $25(\mathrm{OH}) \mathrm{D}_{3}$ half-life (Tables 2 and 3).

The UK study was performed during winter and early spring when vitamin D status is at its lowest. In contrast, there is little seasonal change in the availability of vitamin Dproducing UVB radiation in The Gambia. Consequently, we observed a highly significant difference in $25(\mathrm{OH}) \mathrm{D}$ plasma concentration between countries such that country may act as a surrogate for 25(OH)D in our analysis. Models that examined other predictors of $25(\mathrm{OH}) \mathrm{D}$ and that included country had significantly different intercepts, reflecting the effect of country (probably acting as a proxy for UVB exposure) on $25(\mathrm{OH}) \mathrm{D}$. We did not formally assess skin pigmentation in this study and therefore cannot comment on the effect of differences in skin pigmentation on $25(\mathrm{OH}) \mathrm{D}$ production. $1,25(\mathrm{OH})_{2} \mathrm{D}$ was also a positive predictor of $25(\mathrm{OH}) \mathrm{D}$ and may suggest that the greater supply of $25(\mathrm{OH}) \mathrm{D}$ permits greater renal production of $1,25(\mathrm{OH})_{2} \mathrm{D}$ when calcium intake is low. This was partly unexpected because $25(\mathrm{OH}) \mathrm{D}$ and $1,25(\mathrm{OH})_{2} \mathrm{D}$ do not generally correlate well, except when $25(\mathrm{OH}) \mathrm{D}$ is low [28], although others have reported a correlation across a range of $25(\mathrm{OH}) \mathrm{D}$ plasma concentrations [28, 29]. The other major product of $25(\mathrm{OH}) \mathrm{D}$ is $24,25(\mathrm{OH})_{2} \mathrm{D}$, 
and we found a very strong association between $24,25(\mathrm{OH})_{2} \mathrm{D}$ and its precursor, consistent with previous studies [11, 31]. Multivariate models also indicated weight as a significant positive independent predictor of 25(OH)D. This is in contrast to other studies that have generally shown poorer vitamin D status with increasing adiposity. In our study, participants were relatively lean, and the BMI range was narrow (BMI, $18-27 \mathrm{~kg} / \mathrm{m}^{2}$ ). This may have precluded seeing the inverse relationship reported between 25(OH)D plasma concentration and adiposity [32]. If weight was replaced with fat mass, then no significant association was observed, but with fat-free mass, we observed a similar response to the weight model.

Our study has a number of limitations. It was designed to compare $25(\mathrm{OH}) \mathrm{D}_{3}$ half-life between different populations and its predictors. We restricted participants to men within relatively narrow age and BMI ranges, so we cannot report on how the relationships may be different in women, in different age groups or in overweight and obese individuals. Previous reports of changes in half-life, or strong correlations between half-life and other variables, have primarily been obtained from studies with an acute change, such as a $1,25(\mathrm{OH})_{2} \mathrm{D}$ challenge or a very high-fibre diet, or in a disease state [6-9, 33]. It may be more difficult to detect associations in a small cross-sectional study such as this, especially in free-living individuals. The relatively small sample size limited the power of the statistical methods and means that the findings in this study should be interpreted with a degree of caution. Studies in larger cohorts are required to confirm the predictors of 25(OH)D half-life found here.

In conclusion, this study investigated predictors of the plasma half-life of stable isotope-labelled $25(\mathrm{OH}) \mathrm{D}_{3}$ in freeliving individuals in two different countries. $25(\mathrm{OH}) \mathrm{D}_{3}$ halflife was predicted by factors associated with vitamin $\mathrm{D}$ metabolism $\left(25(\mathrm{OH}) \mathrm{D}, 1,25(\mathrm{OH})_{2} \mathrm{D}\right.$, DBP, plasma phosphate and $\mathrm{PTH}$ ), although an inverse association with $\mathrm{Ca}_{\text {alb }}$ was found. There were differences between countries in factors predicting $25(\mathrm{OH}) \mathrm{D}_{3}$ half-life, and it is perhaps surprising, given the large differences in calcium intake and $1,25(\mathrm{OH})_{2} \mathrm{D}$ plasma concentration, that there was not a significant difference in $25(\mathrm{OH}) \mathrm{D}_{3}$ half-life between countries. This may suggest a degree of adaptation, or regulation of $25(\mathrm{OH}) \mathrm{D}_{3}$ halflife, either through expression of vitamin D hydroxylases or because of genetic differences. In contrast, $25(\mathrm{OH}) \mathrm{D}$ was primarily predicted by country, probably reflecting differences in UVB exposure between the UK in winter and The Gambia. $25(\mathrm{OH}) \mathrm{D}$ half-life has value as a marker of $25(\mathrm{OH}) \mathrm{D}$ expenditure that can disentangle the effects of vitamin $\mathrm{D}$ supply and utilisation. An understanding of factors that affect $25(\mathrm{OH}) \mathrm{D}$ expenditure are required to determine how vitamin $\mathrm{D}$ requirements may vary during the life course and between populations. Opportunities to explore either acute or longer-term interventions that may transiently change $25(\mathrm{OH}) \mathrm{D}$ expenditure should be explored to further enhance the understanding of vitamin D metabolism.

Acknowledgments We thank the participants from Keneba and Cambridge. We also thank MRC staff in Keneba, in particular Buba Sise, Ebrima Sise and Ebrima Danso for field and laboratory work, and Duangporn Harnpanich for coordinating study procedures in Cambridge. Biochemistry analysis was performed by Ann Laidlaw, Janet Bennett and Shailja Nigdikar at MRC HNR. We thank Erik van Herck and Ivo Jans (KU Leuven, Belgium) for plasma DBP and 24,25(OH $)_{2} \mathrm{D}$ analysis.

\section{Conflicts of interest None.}

Funding This research is jointly funded by the Medical Research Council (MRC) (programme codes U105960371, U123261351, MCA760-5QX00) and the Department for International Development (DFID) under the MRC/DFID Concordat agreement.

Open Access This article is distributed under the terms of the Creative Commons Attribution Noncommercial License which permits any noncommercial use, distribution, and reproduction in any medium, provided the original author(s) and the source are credited.

\section{References}

1. Bouillon R, Carmeliet G, Verlinden L, van Etten E, Verstuyf A, Luderer HF, Lieben L, Mathieu C, Demay M (2008) Vitamin D and human health: lessons from vitamin $\mathrm{D}$ receptor null mice. Endocr Rev 29:726-776

2. Gray RW, Weber HP, Dominguez JH, Lemann J Jr (1974) The metabolism of vitamin $\mathrm{D}_{3}$ and 25-hydroxyvitamin $\mathrm{D}_{3}$ in normal and anephric humans. J Clin Endocrinol Metab 39:1045-1056

3. Smith JE, Goodman DS (1971) The turnover and transport of vitamin $D$ and a polar metabolite with the properties of $25-$ hydroxycholecalciferol in human plasma. J Clin Invest 50:21592167

4. Vicchio D, Yergey A, O'Brien K, Allen L, Ray R, Holick M (1993) Quantification and kinetics of 25 -hydroxyvitamin $\mathrm{D}_{3}$ by isotope dilution liquid chromatography/thermospray mass spectrometry. Biol Mass Spectrom 22:53-58

5. Jones KS, Schoenmakers I, Bluck LJ, Ding S, Prentice A (2012) Plasma appearance and disappearance of an oral dose of 25hydroxyvitamin $\mathrm{D}_{2}$ in healthy adults. Br J Nutr 107:1128-1137

6. Clements MR, Davies M, Hayes ME, Hickey CD, Lumb GA, Mawer EB, Adams PH (1992) The role of 1,25-dihydroxyvitamin D in the mechanism of acquired vitamin D deficiency. Clin Endocrinol 37: $17-27$

7. Clements MR, Johnson L, Fraser DR (1987) A new mechanism for induced vitamin D deficiency in calcium deprivation. Nature 325:6265

8. Davies M, Heys SE, Selby PL, Berry JL, Mawer EB (1997) Increased catabolism of 25-hydroxyvitamin D in patients with partial gastrectomy and elevated 1,25-dihydroxyvitamin D levels. Implications for metabolic bone disease. J Clin Endocrinol Metab 82:209-212

9. Batchelor AJ, Compston JE (1983) Reduced plasma half-life of radio-labelled 25-hydroxyvitamin $\mathrm{D}_{3}$ in subjects receiving a highfibre diet. Br J Nutr 49:213-216

10. Jones KS, Schoenmakers I, Assar S, Harnpinach D, Prentice A (2014) $25(\mathrm{OH}) \mathrm{D}_{2}$ half-life is shorter than $25(\mathrm{OH}) \mathrm{D}_{3}$ half-life and is influenced by vitamin $\mathrm{D}$ binding protein concentration and genotype. J Clin Endocrinol Metab 99:3373-3381 
11. Schoenmakers I, Ginty F, Jarjou LM, Nigdikar S, Bennett J, Laidlaw A, Prentice A (2010) Interrelation of parathyroid hormone and vitamin D metabolites in adolescents from the UK and The Gambia. J Steroid Biochem Mol Biol 121:217-220

12. Prynne C, Paul AA (2007) Food composition table for use in The Gambia. MRC Human Nutrition Research, Cambridge

13. Fitt E, Cole D, Ziauddeen N, Pell D, Stickley E, Harvey A, Stephen AM (2014) DINO (Diet In Nutrients Out) - an integrated dietary assessment system. Public Health Nutr DOI:. doi:10.1017/ S1368980014000342

14. Bouillon R, Baelen HV, Moor PD (1977) The measurement of the vitamin D-binding protein in human serum. J Clin Endocrinol Metab 45:225-231

15. Casetta B, Jans I, Billen J, Vanderschueren D, Bouillon R (2010) Development of a method for the quantification of $1 \alpha, 25(\mathrm{OH})_{2}$ vitamin $\mathrm{D}_{3}$ in serum by liquid chromatography tandem mass spectrometry without derivatization. Eur J Mass Spectrom 16:81-89

16. Schoenmakers I, Jarjou LMA, Goldberg GR, Tsoi K, Harnpanich D, Prentice A (2013) Acute response to oral calcium loading in pregnant and lactating women with a low calcium intake: a pilot study. Osteoporos Int 24:2301-2308

17. Abboud M, Puglisi DA, Davies BN, Rybchyn M, Whitehead NP, Brock KE, Cole L, Gordon-Thomson C, Fraser DR, Mason RS (2013) Evidence for a specific uptake and retention mechanism for 25-hydroxyvitamin D (25OHD) in skeletal muscle cells. Endocrinology 154:3022-3030

18. Batchelor AJ, Watson G, Compston JE (1982) Changes in plasma half-life and clearance of ${ }^{3} \mathrm{H}-25$-hydroxyvitamin $\mathrm{D}_{3}$ in patients with intestinal malabsorption. Gut 23:1068-1071

19. Haddock L, Vazquez MC, Rivera R, Corcino J (1985) The kinetics of D3-3H metabolism in tropical sprue. P R Health Sci J 4:47-56

20. Anderson PH, Lee AM, Anderson SM, Sawyer RK, O'Loughlin PD, Morris HA (2010) The effect of dietary calcium on $1,25(\mathrm{OH})_{2} \mathrm{D}_{3}$ synthesis and sparing of serum $25(\mathrm{OH}) \mathrm{D}_{3}$ levels. J Steroid Biochem Mol Biol 121:288-292

21. IOM (2011) Dietary reference intakes for calcium and vitamin D. The National Acadamies, Washington

22. Brown AJ, Dusso A, Slatopolsky E (1999) Vitamin D. Am J Physiol Renal Physiol 277:F157-F175
23. Braithwaite V, Jones KS, Assar S, Schoenmakers I, Prentice A (2014) Predictors of intact and C-terminal fibroblast growth factor 23 in Gambian children. Endocr Connect 3:1-10

24. Gravesen E, Mace ML, Hofman-Bang J, Olgaard K, Lewin E (2014) Circulating FGF23 levels in response to acute changes in plasma $\mathrm{Ca}(2+)$. Calcif Tissue Int 95:46-53

25. Chun RF, Peercy BE, Orwoll ES, Nielson CM, Adams JS, Hewison M (2014) Vitamin D and DBP: the free hormone hypothesis revisited. J Steroid Biochem Mol Biol 144:132-137

26. Bouillon R, van Baelen H, de Moor P (1980) Comparative study of the affinity of the serum vitamin D-binding protein. J Steroid Biochem 13:1029-1034

27. Mawer EB, Schaefer K, Lumb GA, Stanbury SW (1971) The metabolism of isotopically labelled vitamin $\mathrm{D}_{3}$ in man: the influence of the state of vitamin D nutrition. Clin Sci 40:39-53

28. Prentice A, Goldberg GR, Schoenmakers I (2008) Vitamin D across the lifecycle: physiology and biomarkers. Am J Clin Nutr 88:500S$506 \mathrm{~S}$

29. Møller UK, Sv S, Jensen LT, Mosekilde L, Schoenmakers I, Nigdikar S, Rejnmark L (2013) Increased plasma concentrations of vitamin D metabolites and vitamin D binding protein in women using hormonal contraceptives: a cross-sectional study. Nutrients $5: 3470-3480$

30. Vanderschueren D, Pye SR, O'Neill TW et al (2013) Active vitamin D (1,25-dihydroxyvitamin D) and bone health in middle-aged and elderly men: the European Male Aging Study (EMAS). J Clin Endocrinol Metab 98:995-1005

31. Wagner D, Hanwell HE, Schnabl K, Yazdanpanah M, Kimball S, Fu L, Sidhom G, Rousseau D, Cole DEC, Vieth R (2011) The ratio of serum 24,25 -dihydroxyvitamin $\mathrm{D}_{3}$ to 25 -hydroxyvitamin $\mathrm{D}_{3}$ is predictive of 25-hydroxyvitamin $\mathrm{D}_{3}$ response to vitamin $\mathrm{D}_{3}$ supplementation. J Steroid Biochem Mol Biol 126:72-77

32. Young KA, Engelman CD, Langefeld CD, Hairston KG, Haffner SM, Bryer-Ash M, Norris JM (2009) Association of plasma vitamin D levels with adiposity in Hispanic and African Americans. J Clin Endocrinol Metab 94:3306-3319

33. Clements MR, Davies M, Fraser DR, Lumb GA, Mawer EB, Adams PH (1987) Metabolic inactivation of vitamin D is enhanced in primary hyperparathyroidism. Clin Sci 73:659-664 